\title{
Rapid Ambiguity Resolution for Single Frequency GPS Kinematical Positioning Based on Left Null Space Theory
}

\author{
Rong Duan*, Xiubin Zhao, Chunlei Pang, Ang Gong \\ College of Information and Navigation, Air Force Engineering University, Xi'an 710077, China. \\ * Corresponding author. Tel.: +86 13572251600; email: duanrong0919@163.com \\ Manuscript submitted February 29, 2015; accepted May 8, 2015. \\ doi: 10.17706/jcp.11.1.52-61
}

\begin{abstract}
According to the dynamic GPS rapid positioning ambiguity resolution problems, a modified algorithm for rapid integer ambiguity resolution is proposed. Firstly, a method of QR decomposition is applied on the observation matrix to construct the left null space matrix in order to construct the left null space matrix, so as to decrease dimensions of the matrix and to satisfy rapid ambiguity resolution by matrix transformation that can separate out the ambiguity parameter from the position parameter. Kalman filter is applied only to estimate the ambiguity parameters in order to acquire the real-time ambiguity float solution. Then, ordering and multi-time (inverse) paired Cholesky decomposition are adopted for decorrelation of ambiguity. One applies diagonal elements preprocessing, sorts diagonal elements according to the results of Cholesky decomposition, thus to improve the efficiency of decomposition and decorrelation. Lastly, the LAMBDA method is used for searching the fixed integer ambiguity. To verify the correctness and effectiveness of the proposed algorithm, the static and kinematic tests are carried out. Experimental results show that this algorithm has good performance of decorrelation and precision of float solution. It also can effectively improve computation speed. The final positioning accuracy result with static baseline error less than $1 \mathrm{~cm}$ and kinematic error less than $2 \mathrm{~cm}$.The method may be used in areas requiring accurate positioning such as kinematic navigation and geodesy.
\end{abstract}

Key words: GPS, Integer ambiguity, QR decomposition, multi-time (inverse) paired Cholesky decomposition.

\section{Introduction}

Rapid ambiguity resolution is the key problem of GPS carrier phase measurements for high precision real-time positioning [1], [2], which has been studied by many researchers during the last two decades. Examples of proposed methods were dual-frequency P code pseudo range algorithm [3], least squares(LS) searching algorithm [4], ambiguity function algorithm [5], fast ambiguity resolution approach(FARA) [6], Cholesky decomposition [7], OMEGA [8], LAMBDA [9]-[11], etc. Recently, the rapid integer least-squares estimation for high-dimensional ambiguity resolution using lattice theory was proposed [12], [13]. Among them, some were based on dual frequency measurements. When applied for single frequency kinematic positioning, those methods required more epochs, resulting in large amount of calculation caused by higher-order matrix inversion operation and a long time to fix the ambiguity. Thus, those methods could not meet the requirement of real time kinematic applications.

To solve this problem, a new algorithm is proposed to rapid resolve integer ambiguity of kinematic application. Firstly, QR decomposition and transformation are applied to construct a left null space matrix to 
remove baseline coordinate parameters, ambiguity parameters are successfully separated from position parameters. Kalman filter is used to estimate only the ambiguity parameters that acquire real-time float solution of integer ambiguity. Then, diagonal entries of covariance matrix are sorted, and multi-time (inverse) paired Cholesky decomposition is applied for the decorrelation of ambiguity. One applies diagonal elements preprocessing, sorts diagonal elements according to the results of Cholesky decomposition, thus to improve the efficiency of decomposition and decorrelation. Finally, integer solution is fixed by LAMBDA method. Static and kinematic experiments prove the good performance and feasibility of the new algorithm.

\section{Rapid Calculation of Ambiguity Float Solution Based on QR Decomposition}

Let us suppose that base station and mobile station can observe $\mathrm{n}$ satellites synchronously, and each epoch can form $n-1$ double difference carrier phase measurement equations. For the ith epoch, GPS linearized observation equations are generally expressed as

$$
\mathbf{L}_{i}=\mathbf{A}_{i} \delta \mathbf{X}_{i}+\mathbf{B N}+\varepsilon_{i}
$$

where $\mathbf{L}_{i} \in \mathbf{R}^{n-1}$ denotes the observation vector of double difference (DD)carrier phase at the ith epoch, which is the difference between actual value and the calculated one. $\mathbf{A}_{i} \in \mathbf{R}^{(n-1) \times 3}$ is coefficient matrix at the ith epoch. $\delta \boldsymbol{X}_{i}$ is the unknown parameter vector of 3D baseline. $\mathbf{N} \in \mathbf{Z}^{n-1}$ is the unknown DD ambiguity parameters vector with $n-1$ dimensions, which is independent of the epoch. $\mathbf{B}=\operatorname{diag}\left(\lambda_{L_{1}}, \lambda_{L_{1}}, \cdots \lambda_{L_{1}}\right)$ is the coefficient matrix with $n$-1 dimensions, where $\lambda_{L_{1}}$ is the $L_{1}$ carrier wavelength. $\varepsilon_{i}$ is the measurement noise vector.

In rapid positioning, we expect that fewer epochs or even single epoch can realize positioning. According to (1), it has 3 rank deficiencies when computing in single epoch, thus the LS method is unavailable. General method is to increase the number of observation epochs in order to increase equations. For m epochs, relative equations in matrix form are

$$
\mathbf{L}=\mathbf{A} \boldsymbol{\delta} \mathbf{X}+\mathbf{B N}+\varepsilon
$$

High-order matrix inversion is a problem [14] of huge computation when directly solving (2). In order to obtain high precision ambiguity float solution, one needs at least 200 to 400 epochs, which results in very high-order matrix with about 600 to 1200 dimensions. Thus, it can not meet the requirement of real-time kinematic positioning. As we only care about ambiguity float solution and its covariance, this paper applies QR decomposition to coefficient matrix[15]. Baseline component correction vector is eliminated by constructing left null space matrix $\mathbf{L}_{A}$ according to $U$ matrix features. By doing so, ambiguity parameters are successfully separated from position parameters, and matrix dimension is decreased. QR decomposition based transformation steps are showed as follows:

One starts first to solve the left null space matrix of coefficient matrix $\mathbf{A}_{i}$. Since QR decomposition to $\mathbf{A}_{i}$ is carried out, one has $\mathbf{A}_{i}=\mathbf{Q R}$, where $\mathbf{Q}$ is $(n-1) \times(n-1)$ unitary matrix, $\mathbf{R}$ is $(n-1) \times 3$ unitary matrix.

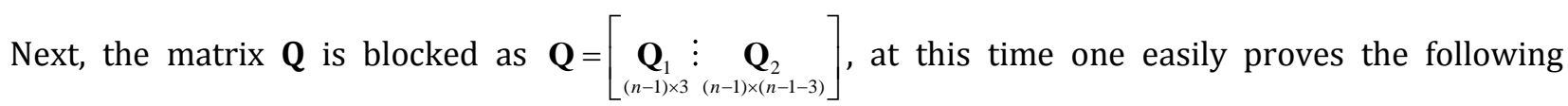
formula: $\mathbf{L}_{A_{i}}=\mathbf{Q}_{2}^{T}$, that is $\mathbf{L}_{A_{i}} \cdot \mathbf{A}_{i}=0$

Finally, Let (2) multiplied by left null space matrix $\mathbf{L}_{A_{i}}$, one will get transformed equation as

$$
\mathbf{L}_{A_{i}} \mathbf{L}_{i}=\mathbf{L}_{A_{i}} \mathbf{B N}+\mathbf{L}_{A_{i}} \varepsilon_{i}
$$

Considering the characteristics that integer ambiguity of each epoch is equal when there is no cycle slip, Kalman filtering state equation and measurement equation [16] are respectively written as 


$$
\begin{gathered}
\mathbf{N}_{k}=\mathbf{N}_{k-1} \\
\mathbf{Z}_{k}=\mathbf{H}_{k} \mathbf{N}_{k}+\mathbf{V}_{k}
\end{gathered}
$$

where $\mathbf{Z}_{k}=\mathbf{L}_{A_{k}} \mathbf{L}_{k}, \mathbf{H}_{k}=\mathbf{L}_{A_{k}} \mathbf{B} \cdot \boldsymbol{V}_{k}$ is the measurement white noise with mean zero and variance $\mathbf{R}_{k}=\operatorname{cov}\left(\mathbf{V}_{k}, \mathbf{V}_{k}\right)=E\left[\mathbf{V}_{k} \mathbf{V}_{k}^{T}\right]=\sigma^{2} \mathbf{L}_{A} \mathbf{L}_{A}^{T}=\sigma^{2} \mathbf{I}$.

Since the state vector of integer ambiguity $N$ is constant value, time update process (prediction process) during Kalman filtering can be simplified as follow

$$
\left\{\begin{array}{l}
\hat{\mathbf{N}}_{k}{ }^{-}=\hat{\mathbf{N}}_{k-1} \\
\mathbf{Q}_{\hat{N} k}{ }^{-}=\mathbf{Q}_{\hat{N} k-1}
\end{array}\right.
$$

Kalman filter Measurement update process (calibration process) are given as

$$
\left\{\begin{array}{l}
\mathbf{K}_{k}=\mathbf{Q}_{\hat{N} k}{ }^{-} \mathbf{H}_{k}{ }^{T}\left[\mathbf{H}_{k} \mathbf{Q}_{\hat{N} k}{ }^{-} \mathbf{H}_{k}{ }^{T}+\mathbf{R}_{k}\right]^{-1} \\
\hat{\mathbf{N}}_{k}{ }^{+}=\hat{\mathbf{N}}_{k}{ }^{-}+\mathbf{K}_{k}\left(\mathbf{Z}_{k}-\mathbf{H}_{k} \hat{\mathbf{N}}_{k}{ }^{-}\right) \\
\mathbf{Q}_{\hat{N} k}{ }^{+}=\left[\mathbf{I}-\mathbf{K}_{k} \mathbf{H}_{k}\right] \mathbf{Q}_{\hat{N} k}^{-}
\end{array}\right.
$$

Among (7), $\mathbf{K}_{k}$ is filtering gain, $\mathbf{I}$ is unit matrix. According to the initial value of state vector $\hat{\mathbf{N}}_{0}$ and the estimation error covariance $\mathbf{Q}_{\hat{N} 0}$, we can compute the optimal estimation of ambiguity state vector and estimation error covariance at any time. Filtering equations indicate that position parameters and velocity parameters are eliminated during the process of calculation. Obviously, the amount of computation is greatly reduced due to the avoiding of high-order matrix inversion, which can do a good help to ambiguity estimation in real-time kinematic applications.

\section{Sorting and Multi-time (Inverse) Paired Cholesky Decomposition for Decorrelation}

Real-time float solution of ambiguity can be calculated based on QR decomposition and Kalman filtering. But in actual kinematic positioning environment, during a shot period of observing time, the geometric structure of satellites in view is poor, which leads to strong correlation between DD measurements. This strong correlation makes multi-dimensional ellipsoid search space much longer. Thus, integer ambiguity search results are not as good as expected. To solve this problem, the covariance matrix of float solution needs to be factorized to make it to be diagonalization as much as possible. By doing so, the correlation between ambiguities of DD phase measurements can be reduced, which makes the search ellipsoid space closer to sphere, minimizing length of search interval and improving the search efficiency of integer ambiguity fixing.

Concerning that Gauss transformation for decorrelation is not stable, and its calculation amount is double of Cholesky decomposition. This paper proposes a method of continuously implementing modified upper triangular Cholesky decomposition and lower triangular Cholesky decomposition to realize continuous decorrelating [17]-[19]. Before Cholesky decomposition, sorting the diagonal elements of ambiguity covariance matrix(ascending or descending) can reduce condition number of matrix, effectively improve the performance of matrix decomposition [20]-[22]. Compared to this traditional method, we apply diagonal elements preprocessing. Sorting based on the result of Cholesky decomposition, bigger diagonal entry of UDUT result is adjusted to a smaller $i$ position in diagonal line, LDLT result is on the contrary. After this, numerical size of each diagonal element is much closer to one another. Thus, the validity of matrix decomposition is improved, so is the decorrelation. Then, the efficiency and quality of ambiguity discrete search is improved at last. The steps of sorting and multiple (inverse) paired Cholesky decomposition are as 
follows:

(1) Modified integer UDUT decomposition

1) $\operatorname{Remark} \mathbf{J}_{0}=\mathbf{Q}_{\hat{N}}=\left[q_{i j}\right]_{n \times n}, i=1,2, \cdots, n$, Perform the following steps to $\mathbf{J}_{0}$ row by row

a) Pre-compute candidate elements

$$
\left\{\begin{array}{l}
c_{p j}=\left(q_{p j}-\sum_{k=j+1}^{n} c_{p k} d_{k} c_{j k}\right) / d_{j}, i<j \\
v_{p}=q_{p p}-\sum_{k=i+1}^{n} c_{p k}^{2} d_{k}, p=i, i+1, \cdots, n
\end{array}\right.
$$

In (8), $c_{p j}$ denotes the candidate entry in unit upper triangular matrix $\mathbf{U}, v_{p}$ denotes the candidate in diagonal matrix D.

b) Select the element $v_{i}$ to meet $v_{i}=\max _{i \leq p \leq n}\left\{v_{p}\right\}$, and its index number $M_{i}=p$. So let $u_{i j}=c_{M j}(i<j)$, $d_{i}=v_{M_{i}}, u_{i j}, d_{i}$ denotes the elements in modified Cholesky decomposition $\mathrm{U}$ and $\mathrm{D}$ respectively.

c) Adjust variance-covariance matrix according to the index number from the last step: $\mathbf{J}_{i}=\mathbf{S}_{i}\left(i, M_{i}\right) \mathbf{J}_{i-1} \mathbf{S}_{i}\left(i, M_{i}\right)^{T}$. Where $\mathbf{S}_{i}\left(i, M_{i}\right)$ is adjusting matrix, obtained by exchange of ith row and $M_{i}$ th row in unit matrix $I_{n \times n}$. Especially, when $i=M_{i}, \quad \mathbf{S}_{i}\left(i, M_{i}\right)=\mathbf{I}_{n \times n}$.

2) Modified UDUT decomposition is obtained by the previous step, that is $\mathbf{U}=\left[u_{i j}\right]_{n \times n}$, $\boldsymbol{D}=\operatorname{diag}\left(d_{1}, d_{2}, \cdots, d_{n}\right)$, then one can get that $\mathbf{J}_{n}=\mathbf{U D U} \mathbf{U}^{T}, \quad \mathbf{J}_{n}=\hat{\mathbf{S}} \mathbf{Q}_{\hat{N}} \hat{\mathbf{S}}^{T}, \quad$ where $\hat{\mathbf{S}}=\mathbf{S}_{n}\left(n, M_{n}\right) \mathbf{S}_{n-1}\left(n-1, M_{n-1}\right) \cdots \mathbf{S}_{1}\left(1, M_{1}\right)$.

3) Let entries in upper triangular be integers, calculate the transformation matrix $[\boldsymbol{U}]_{1}^{-1}$ by matrix inversion, then the covariance matrix after modified UDU ${ }^{\mathrm{T}}$ transformation is

$$
\mathbf{Q}_{\hat{u}}=[\mathbf{U}]_{1}^{-1} \boldsymbol{J}_{n}[\mathbf{U}]_{1}^{-T}=[\mathbf{U}]_{1}^{-1} \hat{\mathbf{S}}_{1} \mathbf{Q}_{\hat{N}} \hat{\mathbf{S}}_{1}^{T}[\mathbf{U}]_{1}^{-T}
$$

(2) Modified integer LDLT decomposition

1) $\operatorname{Remark} \mathbf{J}_{0}{ }^{\prime}=\mathbf{Q}_{\hat{u}}=\left[q_{i j}\right]_{n \times n}{ }^{\prime}, i=1,2, \cdots, n$, Perform the following steps to $\mathbf{J}_{0}{ }^{\prime}$ row to row.

a) Pre-compute candidate elements

$$
\left\{\begin{array}{l}
f_{p j}=\left(q_{p j}-\sum_{k=1}^{j-1} f_{p k} d_{k} f_{j k}\right) / d_{j}, i>j \\
v_{p}=q_{p p}-\sum_{k=1}^{j-1} f_{p k}^{2} d_{k}, p=i, i+1, \cdots, n
\end{array}\right.
$$

In (10), $f_{p j}$ is the candidate element in unit lower triangular matrix $\mathbf{L}, v_{p}$ denotes the candidate element in diagonal matrix $\mathbf{D}$.

b) Select element $v_{i}$ to meet $v_{i}=\min _{i \leq p \leq n}\left\{v_{p}\right\}$, and its index number $M_{i}=p$. So let $l_{i j}=f_{M j}(i>j)$, $d_{i}=v_{M_{i}}, l_{i j}, d_{i}$ denotes the elements in modified Cholesky decomposition $\mathbf{L}$ and $\mathbf{D}$ respectively.

c) Adjust variance-covariance matrix according to the index number from the last step: $\mathbf{J}_{i}^{\prime}=\mathbf{G}_{i}\left(i, M_{i}\right) \mathbf{J}_{i-1}{ }^{\prime} \mathbf{G}_{i}\left(i, M_{i}\right)^{T}$. Where $\mathbf{G}_{i}\left(i, M_{i}\right)$ is adjusting matrix, obtained by exchange of ith row and $M_{i}$ th row in unit matrix $I_{n \times n}$. Especially, when $i=M_{i}, \quad \mathbf{G}_{i}\left(i, M_{i}\right)=\mathbf{I}_{n \times n}$. 
2) Modified $\mathrm{LDL}^{\mathrm{T}}$ decomposition is obtained by the previous step, that is $\mathbf{L}=\left[l_{i j}\right]_{n \times n}, \quad \mathbf{D}=\operatorname{diag}\left(d_{1}, d_{2}, \cdots, d_{n}\right)$ , then one can get that $\boldsymbol{J}_{n}{ }^{\prime}=\boldsymbol{L} \boldsymbol{D} \boldsymbol{L}^{T}, \quad \boldsymbol{J}_{n}{ }^{\prime}=\hat{\boldsymbol{G}} \boldsymbol{Q}_{\hat{u}} \hat{\boldsymbol{G}}^{T}$, where $\hat{\mathbf{G}}=\mathbf{G}_{n}\left(n, M_{n}\right) \mathbf{G}_{n-1}\left(n-1, M_{n-1}\right) \cdots \mathbf{G}_{1}\left(1, M_{1}\right)$.

3) Let entries in lower triangular be integers, calculate the transformation matrix $[\mathbf{L}]_{1}^{-1}$ by matrix inversion, then the covariance matrix after modified $\mathrm{LDL}^{\mathrm{T}}$ transformation is

$$
\begin{aligned}
\mathbf{Q}_{\hat{N}}= & {[\mathbf{L}]_{1}^{-1} \boldsymbol{J}_{n}^{\prime}[\mathbf{L}]_{1}^{-T}=[\mathbf{L}]_{1}^{-1} \hat{\mathbf{G}}_{1} \mathbf{Q}_{\hat{u}} \hat{\mathbf{G}}_{1}^{T}[\mathbf{L}]_{1}^{-T}=} \\
& \left\{[\mathbf{L}]_{1}^{-1} \hat{\mathbf{G}}_{1}[\mathbf{U}]_{1}^{-1} \hat{\boldsymbol{S}}_{1} \mathbf{Q}_{\hat{N}} \hat{\boldsymbol{S}}_{1}^{T}[\mathbf{U}]_{1}^{-T} \hat{\mathbf{G}}_{1}^{T}[\mathbf{L}]_{1}^{-T}\right\}
\end{aligned}
$$

(3) Check whether A is the identity matrix. If so, then end the calculation; otherwise, it show that the correlation between the undetermined ambiguity is quite strong, return to step (1) carrying on loop computing until $[\mathbf{L}]_{1}^{-1}$ becomes identity matrix, so far, now stop decomposition.

Perform the modified integer UDUT decomposition and $\mathrm{LDL}^{\mathrm{T}}$ decomposition repeatedly. Assuming that iteration is executed for $m$ times, when $[\mathbf{L}]_{1}^{-1}$ is transformed to be a unit matrix, the final transformation matrix is going to be

$$
\begin{aligned}
\mathbf{T}_{z}= & \left([\mathbf{L}]_{m}^{-1} \hat{\mathbf{G}}_{m}[\mathbf{U}]_{m}^{-1} \hat{\mathbf{S}}_{m}\right) \cdot\left([\mathbf{L}]_{m-1}^{-1} \hat{\mathbf{G}}_{m-1}[\mathbf{U}]_{m-1}^{-1} \hat{\mathbf{S}}_{m-1}\right) \cdot \\
& \cdots \cdot\left([\mathbf{L}]_{1}^{-1} \hat{\mathbf{G}}_{1}[\mathbf{U}]_{1}^{-1} \hat{\mathbf{S}}_{1}\right) \\
= & \prod_{i=1}^{m}\left([\mathbf{L}]_{i}^{-1} \hat{\mathbf{G}}_{i}[\mathbf{U}]_{i}^{-1} \hat{\mathbf{S}}_{i}\right)
\end{aligned}
$$

Accordingly, the covariance matrix of ambiguity after the transformation is

$$
\mathbf{Q}_{\hat{z}}=\mathbf{T}_{z} \mathbf{Q}_{\hat{N}} \mathbf{T}_{z}^{\mathrm{T}}
$$

Integer ambiguity float solution is

$$
\hat{\mathbf{z}}=\mathbf{T}_{z} \hat{\mathbf{N}}
$$

Finally, integer ambiguity is fixed by LAMBDA algorithm. $\mathbf{z}$ is searched using (15) to minimize the object function as the fixed $\hat{\mathbf{z}}$

$$
\min _{z}(\hat{\mathbf{z}}-\mathbf{z})^{\mathrm{T}} \mathbf{Q}_{\hat{z}}^{-1}(\hat{\mathbf{z}}-\mathbf{z})
$$

Then perform inverse transformation

$$
\hat{\mathbf{N}}=\mathbf{T}_{z}^{-1} \hat{\mathbf{z}}
$$

That is the original ambiguity fixed solution.

\section{Experimental Analysis}

In order to verify the accuracy, correctness and effectiveness of proposed algorithm, static and kinematic experiments are carried out respectively. Actual collected receiver data is processed to calculate the navigation result. Then, compare it with the standard value to prove the reliability of this algorithm.

\subsection{Static Test and Result Analysis}

In order to validate the correctness and feasibility, static test was carried out. The data acquisition time is ten thirty-one on May 16, 2013, as the place of Air Force Engineering University Taoyuan campus laboratory building. We used two NovAtel receivers with type OEM628 as one base station and another mobile station, 
GPS-702-GG GPS dual-frequency antenna. Data was collected in half an hour. Baseline between tow receivers is $7.812 \mathrm{~m}$. There were 7 stars in view above elevation $10^{\circ}$, PRN6, PRN8, PRN11, PRN15, PRN17, PRN24, and PRN28. In order to reduce other errors, PRN24 with maximal elevation was chosen as the reference satellite. Actual data was processed by proposed algorithm in simulation, after 128 epoches, ambiguity was fixed. Then, fixed ambiguity was returned to compute the value of baseline to make a comparison with the real value $7.812 \mathrm{~m}$. Fig. 1 is the length of baseline and its error. As can be seen from the figure baseline length error of proposed new algorithm is less than $1 \mathrm{~cm}$, which indicates some good performance of accuracy.

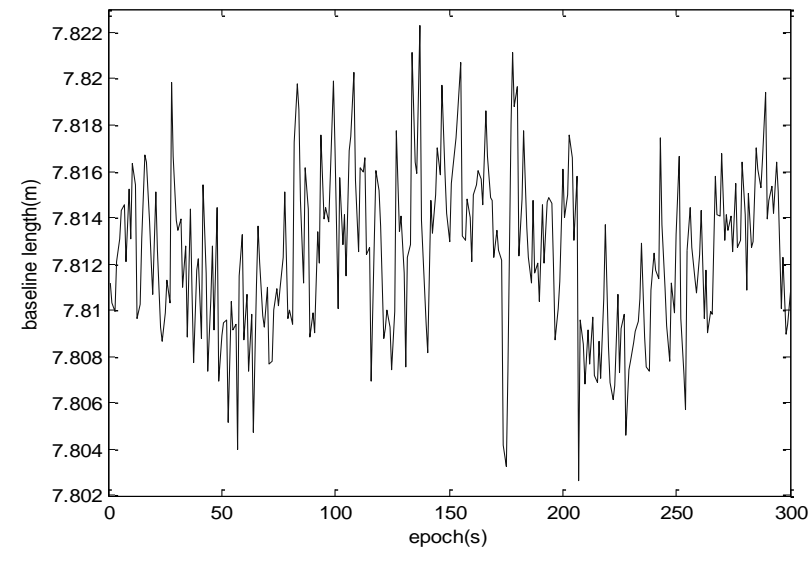

(a) Baseline length

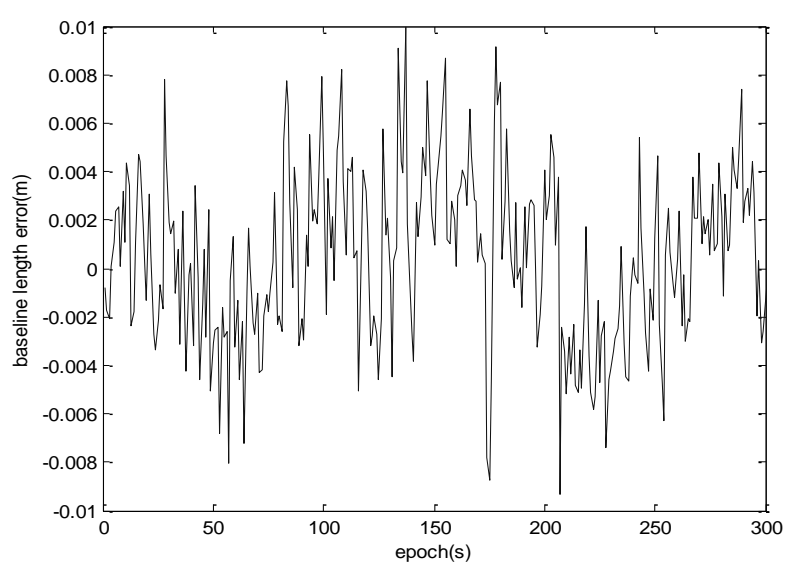

(b) Baseline length error

Fig. 1. Baseline length and its error.

\subsection{Kinematic Test and Result Analysis}

To further validate the applicability of the algorithm in a kinematic environment and a long baseline situation, a vehicle test in a longer distance was carried out.

\subsubsection{Verification scheme}

Using the same test conditions with static test, as due to the dynamic changes of baseline with a much longer distance, the accuracy of the algorithm can not be validated directly, so here by way of dual mobile station, as shown in Fig. 2. Base station is placed below the Lab building in open area, two mobile stations are placed on the top vehicle, one is at the head and another one is at the rear, $2.115 \mathrm{~m}$ apart. After a short period of stop, the vehicle began to go around in 200meters away. Original measurements data and epoch data (L1 frequency) is collected and it was processed by the proposed rapid new algorithm one epoch to another.

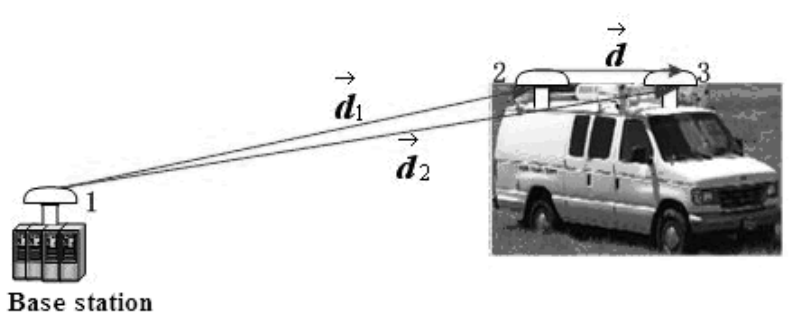

Fig. 2. Sketch of algorithm validation embodiment.

As shown in Fig. 2, the baseline between antenna2 and antenna1 is computed , recorded as $\overrightarrow{\mathbf{d}}_{1}$, so was the baseline between antenna 3 and antenna1, recoded as $\overrightarrow{\mathbf{d}}_{2}$. The baseline between antena 2 and antena3 is fixed and recoded as $\overrightarrow{\mathbf{d}}$. Easy to know $\overrightarrow{\mathbf{d}}=\overrightarrow{\mathbf{d}}_{2}-\overrightarrow{\mathbf{d}}_{1}$. According to the solution of $\overrightarrow{\mathbf{d}}_{1}$ and $\overrightarrow{\mathbf{d}}_{2}$, we can get 
that $|\overrightarrow{\mathbf{d}}|=\left|\overrightarrow{\mathbf{d}}_{1}-\overrightarrow{\mathbf{d}}_{2}\right|$, and then compared it with fixed length of baseline $\mathbf{d}$ to verify the accuracy of relative position.

\subsubsection{Test results analysis}

Using the proposed algorithm, the integer ambiguity between mobile station 2 and the base station is computed, so is the integer ambiguity between mobile station 2 and the base station. Integer ambiguities are rapid fixed when epoch number is 193 and 197 respectively, and Kalman filter tends to be stable. This is because that new algorithm baseline parameters are eliminated by the construct of left null matrix and only ambiguity status vector is concerned during filtering processing, thereby the dimensions of matrix are reduced, and the calculating accuracy and speed of float solution is effectively improved. According to the above-mentioned scheme, the relative position between mobile station 2 and station 3 is computed and the length and error of baseline are shown in Fig. 3(a), the elevation and azimuth of baseline are shown in Fig. $3(b)$.
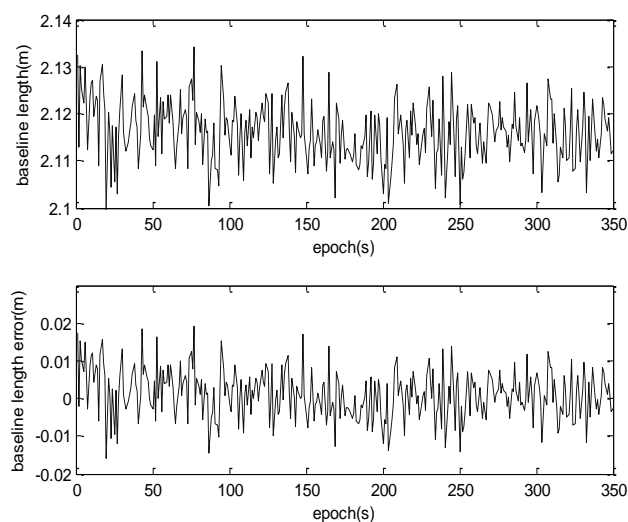

(a) Baseline length and its error
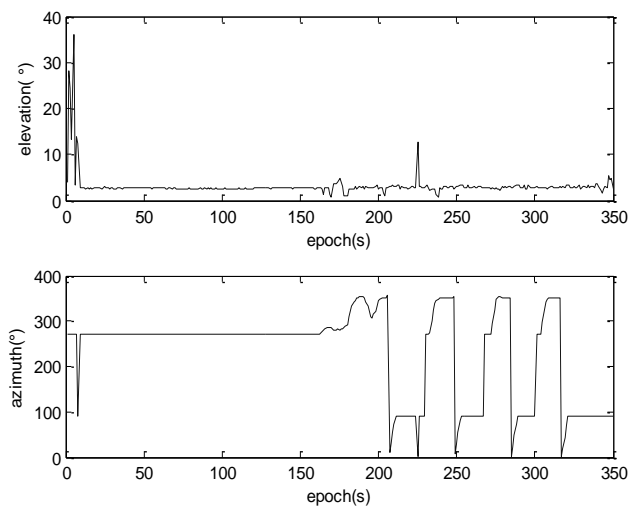

(b) The elevation and azimuth of baseline

Fig. 3. Relative position of the mobile station 2 and station 3.

Table 1. The $r$ and $e$ of Ambiguity Covariance Matrix before and after Decorrelation

\begin{tabular}{ccccc}
\hline \multirow{2}{*}{ Epoch } & \multicolumn{2}{c}{ Before decorrelation } & \multicolumn{2}{c}{ After decorrelation } \\
\cline { 2 - 5 } & $\begin{array}{c}\text { Decorrelation } \\
\text { coefficient } r\end{array}$ & $\begin{array}{c}\text { Spectral condition } \\
\text { number } e\end{array}$ & $\begin{array}{c}\text { Decorrelation } \\
\text { coefficient } r\end{array}$ & $\begin{array}{c}\text { Spectral condition } \\
\text { number } e\end{array}$ \\
\hline 1 & $9.216 \times 10^{-8}$ & 301.225 & 0.622 & 3.356 \\
2 & $9.327 \times 10^{-8}$ & 300.037 & 0.623 & 3.334 \\
3 & $9.399 \times 10^{-8}$ & 298.964 & 0.625 & 3.318 \\
4 & $9.473 \times 10^{-8}$ & 297.993 & 0.625 & 3.305 \\
5 & $9.560 \times 10^{-8}$ & 297.101 & 0.627 & 3.293 \\
6 & $9.634 \times 10^{-8}$ & 296.098 & 0.629 & 3.282 \\
7 & $9.711 \times 10^{-8}$ & 295.252 & 0.630 & 3.302 \\
8 & $9.789 \times 10^{-8}$ & 294.307 & 0.631 & 3.319 \\
9 & $9.873 \times 10^{-8}$ & 293.579 & 0.632 & 3.332 \\
10 & $9.961 \times 10^{-8}$ & 292.626 & 0.632 & 3.346 \\
11 & $1.005 \times 10^{-7}$ & 291.633 & 0.633 & 3.355 \\
12 & $9.731 \times 10^{-7}$ & 290.784 & 0.634 & 3.336 \\
13 & $9.844 \times 10^{-7}$ & 289.851 & 0.635 & 3.318 \\
14 & $9.928 \times 10^{-7}$ & 289.008 & 0.636 & 3.331 \\
15 & $1.003 \times 10^{-7}$ & 288.132 & 0.636 & 3.314 \\
16 & $1.016 \times 10^{-7}$ & 287.156 & 0.637 & 3.303 \\
17 & $1.028 \times 10^{-7}$ & 286.017 & 0.637 & 3.287 \\
18 & $1.034 \times 10^{-7}$ & 285.320 & 0.638 & 3.302 \\
19 & $1.047 \times 10^{-7}$ & 284.464 & 0.638 & 3.313 \\
20 & $1.060 \times 10^{-7}$ & 283.511 & 0.639 & 3.325 \\
\hline
\end{tabular}


As shown in Fig. 3, the computed position relationship between mobile station and base station is consistent with the reality. The errors of elevation and azimuth are stable in a small angle because of the circle motion of vehicle. During the test, when signal quality is good, baseline error is confined in $2 \mathrm{~cm}$, which indicates that ambiguity is correctly fixed. But in some small part of time, error may become larger due to other causes. As the length of baseline, elevation and azimuth are all computed based on the relative position between mobile stations, so this paper makes the length of baseline as criterion is practical and scientific. It further verifies the correctness of the algorithm.

Finally, we analysis the performance of decorrelation, adopting decorrelation coefficient $r$ and spectral condition number $e$ as standard to evaluate the performance of decorrelation [22]. Table 1 shows the decorrelation and spectral condition number before and after decorrelation. As shown in Table 1, decorrelation coefficient $r$ is near 0 before decorrelation, which indicates strong correlation between ambiguities. The spectral condition number $e$ is very large before decorrelation, which indicates a flat search space, also reflecting the strong correlation. After decorrelation, decorrelation coefficient $r$ becomes larger and approaches 1 . While it's spectral condition number e is greatly reduced and covariance matrix is closer to diagonal one. Correlation between different ambiguities is reduced successfully, which indicates the feasibility and good performance of decorrelation of the new algorithm.

\section{Conclusion}

1) Through static and kinematic test and analysis show that the proposed algorithm for ambiguity rapid resolution in this paper is feasible, the static baseline error is less than $1 \mathrm{~cm}$, kinematic baseline error is less than $2 \mathrm{~cm}$, which verifies the correctness and effectiveness of the algorithm.

2) QR decomposition is applied to construct the left null space of matrix to eliminate the baseline coordinate parameters which can separate out the ambiguity parameters from the position parameters. Thus Kalman filter is used to estimate only the ambiguity parameters in the new algorithm, which greatly reduces the amount of computation. Computation speed and real-time capability are both well improved.

3) Sorting and multiple (inverse) Cholesky decompositions are performed for ambiguity decorrelation, adopting method of diagonal entries pre-processing and adjusting the order of diagonal entries according to Cholesky decompositions values. The effectiveness of matrix decomposition is well ensured and much smaller conditional number is obtained, thereby performance of decorrelation is improved, which is good for ambiguity search efficiency and correctness.

As the new algorithm in this paper can realize integer ambiguity rapid solution, acquiring high accurate position quickly, it could be used for 'BDS-2' in some years. It may have a broad application prospects for airborne platform rapid positioning and attitude determination and for precision approach and landing system. It also needs further improvement in some specific engineering implementation.

\section{Acknowledgment}

This work is supported by China National Natural Science Foundation of China (No: 61273049). Special thanks go to anonymous reviewers whose comments have contributed to the improvement of the paper.

\section{References}

[1] Hofmann-Wellenhoff, B., Lichtenegger, H., \& Collins, J. (2001). Global Positioning System: Theory and Practice (5th ed.). Berlin: Springer-Verlag.

[2] Leick, A. (2004). GPS Satellite Surveying (3rd ed.). New York: Wiley.

[3] Blewitt, G. (1989). Carrier phase ambiguity resolution for the global positioning system applied to 
geodetic baselines up to 2000 km. Journal of Geophysical Research, 94(B8), 10187-10203.

[4] Hatch, R. (1990). Instantaneous ambiguity resolution. Proceedings of IAG International Symposium: No. 107 (pp. 99-308). Banff, Alberta, Cananda,.

[5] Remondi, B. W. (1991). Pseudo-kinematic GPS results using the ambiguity function method. Journal of Navigation, 42(1), 109-165.

[6] Frei, E., \& Beulter, G. (1990). Rapid static positioning based on the fast ambiguity resolution approach FARA: Theory and First Results. Manuscripta Geodaetica, 15(6), 325-356.

[7] Euler, H. J., \& Landau, H. (1992). Fast GPS ambiguity resolution on-the-fly for real-time application. Proceedings of Sixth International Geodetic Symposium on Satellite Positioning (pp. 17-20).

[8] Kim, D., \& Langley, R. (1999). An optimized least-squares technique for improving ambiguity resolution and computational efficiency. Proceedings of ION GPS (pp. 1579-1588).

[9] Teunissen, P. J. G. (1994). A new method for fast carrier phase ambiguity estimation. Proceedings of IEEE Position Location and Navigation Symposium PLANS'94 ( pp. 562-573).

[10] Teunissen, P. J. G. (1995). The least-squares ambiguity decorrelation adjustment: a method for fast GPS integer ambiguity estimation. Journal of Geodesy, 70, 65-82.

[11] Teunissen, P. J. G., \& Verhagen, S. (2008). GNSS ambiguity resolution: When and how to fix or not to fix? Proceedings of A Symposium on Theoretical and Computational Geodesy (pp. 143-148).

[12] Jazaeri, S., Amiri-Simkooei, A. R., \& Sharifi, M. A. (2012). Fast integer leastsquares estimation for GNSS high-dimensional ambiguity resolution using lattice theory. Journal of Geodesy, 86(2), 123-136.

[13] Jazaeri, S., Amiri-Simkooei, A., \& Sharifi, M. A. (2014). On lattice reduction algorithms for solving weighted integer least squares problems: comparative study. GPS Solutions, 18(1), 105-114.

[14] Liu, L. L., Liu, J. Y., et al. (2005). Rapid Ambiguity Resolution On-the-Fly for Single Frequency Receiver. Geomatics and Information Science of Wuhan University, 30(10), 885-887.

[15] Timothy, S. (2010). Numerical Analysis. Beijing: Posts \& Telecom Press.

[16] Tomoji, T., \& Akio, Y. (2010). Kalman-filter-based integer ambiguity resolution strategy for long-baseline RTK with ionosphere and troposphere estimation. Proceedings of ION GNSS 2010 (pp. 161-171).

[17] Xu, P. L. (2001). Random simulation and GPS decorrelation. Journal of Geodesy, 75(7), 408-423.

[18] Zhou, Y. M. (2011). A new practical approach to GNSS high-dimensional ambiguity decorrelation. GPS Solutions, 15(4), 325-331.

[19] Zhou, Y. M., \& He, Z. B. (2014). Variance reduction of GNSS ambiguity in (inverse) paired Cholesky decorrelation transformation. GPS Solutions, 18, 509-517.

[20] Liu, L. T., Su, H. T., \& Zhu, Y. Z. (1999). A new approach to GPS ambiguity decorrelation. Journal of Geodesy, 73(6), 478-490.

[21] Chen, S. X., \& Wang, Y. S. (2002). New algorithm for GPS ambiguity decorrelation. Acta Aeronautica Et Astronautica Sinica, 23(6), 542-546.

[22] Huang, Z. Y., \& Chen, S. J. (2010). Modified GPS ambiguity white filtering algorithm. Journal of Southwest Jiaotong University, 45(1), 150-155.

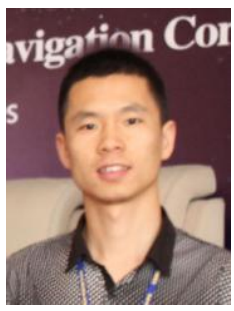

Rong Duan was born in Hunan, China in 1986. He received his B.S. and M.S. degrees from Institute of Telecommunication Engineering, Air Force Engineering University, China, in 2009 and 2012, respectively. Currently, he is an Ph.D. candidate in College of Information and Navigation, Air Force Engineering University, China. His research interests are focused on high accuracy navigation algorithms for close proximity applications such as airplane 
approach and landing, cycle ambiguity resolution, and robust estimation techniques.

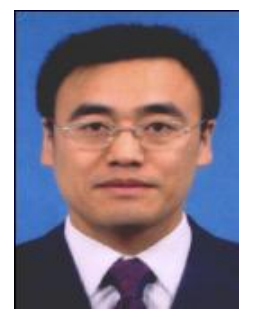

Xiubin Zhao received the M.S. degree in electrical information engineering from Nanjing University of Aeronautics and Astronautics, Nanjing, China, in 1996, the Ph.D. degree in signal and information processing from the Xibei Industry University, Xi'an, China, in 2000. He has been engaged in basic theory research and technology innovation of radio navigation since 1990. He was recruited as a senior member of Chinese Institute of Electronics in 2000, employed as an electronic journal reviewer in 2001, recruited as the national science foundation reviewer in 2003, recruited as a Sahan'xi Provincial Institute of Electronics Radar and Navigation professional member in 2005. His research interests include radio navigation, navigation signal processing, satellite positioning technology and simulation technology.

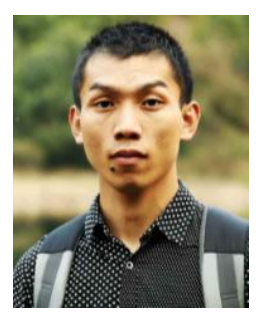

Chunlei Pang received the B.S. degree from Air Force Engineering University, China, in 2007, the M.S. and Ph.D. degrees from same university, in 2010 and 2013. Now he is a research associate in Air Force Engineering University, China. His research interests include GNSS data processing, relative navigation and attitude determination in space and air.

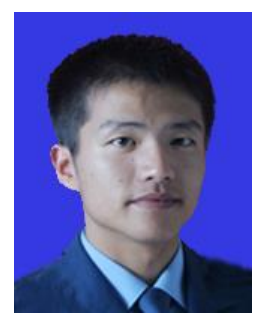

Ang Gong received his B.S. and M.S. degrees from Institute of Telecommunication Engineering, Air Force Engineering University, China, in 2010 and 2013, respectively. Currently, he is an Ph.D. candidate in College of Information and Navigation, Air Force Engineering University, China. His current research interests include GNSS data processing and its application. 